\title{
Antibiotika zur Behandlung von Infektionen durch Methicillin-resistente Staphylococcus aureus (MRSA)*
}

\author{
Antibiotics for Treatment of Infections by Methicillin-resistant \\ Staphylococcus aureus (MRSA)
}

Autor

Institut

\section{R. Stahlmann}

Institut für Klinische Pharmakologie und Toxikologie, Charité - Universitätsmedizin Berlin eingereicht 26.3.2014 akzeptiert nach Revision 7.7.2014

Bibliografie

DOI http://dx.doi.org/ 10.1055/s-0034-1377747 Pneumologie 2014; 68: 676-684 (c) Georg Thieme Verlag KG Stuttgart · New York ISSN 0934-8387

Korrespondenzadresse Prof. Dr. med. Ralf Stahlmann Institut für Klinische Pharmakologie und Toxikologie Charité - Universitätsmedizin Berlin

Luisenstr. 7

10117 Berlin

ralf.stahlmann@charite.de

\section{Zusammenfassung \\ $\nabla$}

In den vergangenen 50 Jahren haben sich die Methicillin-resistenten S. aureus-Stämme (MRSA) weltweit verbreitet. Nach wie vor ist Vancomycin das überwiegend empfohlene Antibiotikum zur Behandlung entsprechender Infektionen. Teicoplanin stellt eine Alternative mit längerer Halbwertzeit dar. Telavancin ist ein neueres Vancomycin-Derivat, das in klinischen Studien etwa gleich wirksam wie Vancomycin war. Bei Patienten mit Niereninsuffizienz sollte es nicht angewandt werden. Nephrotoxische Wirkungen der Glykopeptide schränken ihre therapeutische Verwendbarkeit ein. Das Oxazolidinon Linezolid ist dem Vancomycin therapeutisch gleichwertig bis überlegen. Bei einer Therapie mit dieser Substanz sind Blutbildkontrollen notwendig, neurotoxische Wirkungen wurden vor allem bei längerer Behandlungsdauer beobachtet. Beachtet werden müssen die Interaktionen mit serotoninerg wirksamen Arzneistoffen. Neue Möglichkeiten eröffnen sich mit Ceftarolin, dem ersten MRSA-wirksamen $\beta$-Laktamantibiotikum. Kontrollierte Studien bei pulmonalen MRSA-Infektionen liegen allerdings nicht vor. Daptomycin, ein Lipopeptid, und Tigecyclin, ein Glycylcyclin, zeigen in vitro ebenfalls Aktivität gegen MRSA, kommen aber zur Behandlung pulmonaler MRSA-Infektionen ebenfalls nicht in Betracht. Am Beispiel dieser Antibiotika wird deutlich, dass der Nachweis einer in vitro-Aktivität zwar von Bedeutung ist, dass aber die für eine Therapieentscheidung notwendigen Erkenntnisse nur aus doppelblind-randomisierten klinischen Studien abgeleitet werden können.

\section{Abstract \\ $\nabla$}

Over the last 50 years methicillin-resistant S. aureus (MRSA) spread globally. Vancomycin is still the most recommended antibiotic for MRSA-infections. Teicoplanin is an alternative glycopeptide with longer elimination half-life. Telavancin is a more recently developed derivative of vancomycin with similar clinical efficacy as vancomycin. It is not recommended for treatment of patients with renal insufficiency. Nephrotoxicity limits the therapeutic use of glycopeptide antibiotics. The oxazolidinone linezolid exhibits similar to superior therapeutic efficacy. Hematologic controls are necessary during treatment with this antibacterial agent. Neurotoxic effects have been observed mainly in patients who received prolonged linezolid treatment. Attention must be paid to possible interactions with concomitantly given drugs acting on the serotonergic system. New therapeutic options arise with ceftaroline, the first $\beta$-lactam antibiotic with activity against MRSA. However, controlled clinical trials with pulmonary MRSA infections have not been conducted with ceftaroline. Daptomycin, a lipopeptide, and tigecycline, a glycylcyclin are active in vitro against MRSA as well, but are also not indicated in pulmonary MRSA infections. These antibiotics show in an exemplary manner that antibacterial activity in vitro is an important prerequisite, but relevant data for a therapeutic decision should be derived from randomized controlled clinical double-blind trials.

\footnotetext{
* Professor Hartmut Lode zum 75. Geburtstag gewidmet.
} 


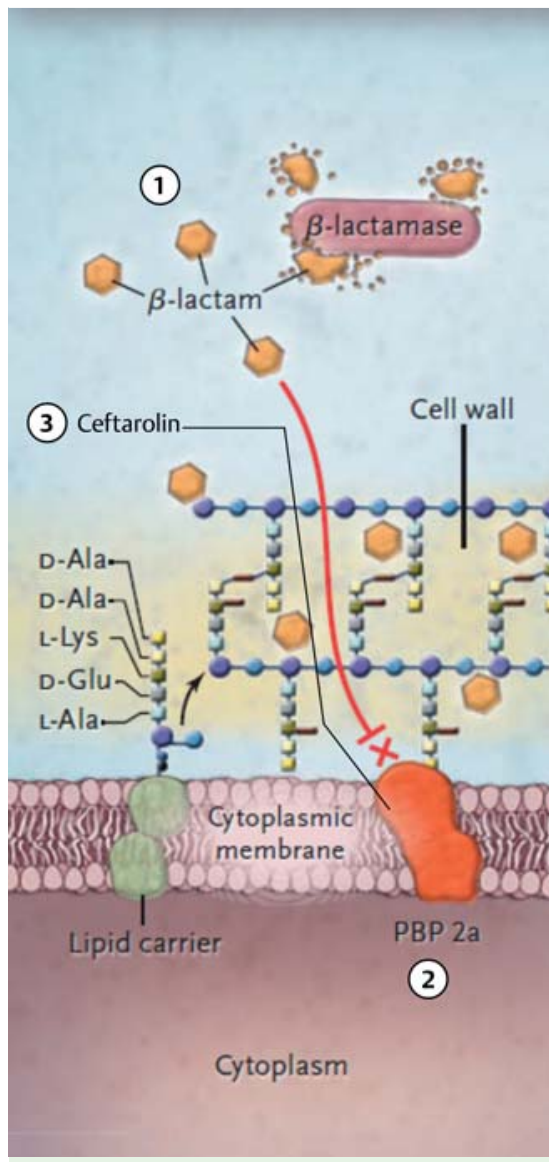

Abb. 1 ß-Laktamresistenz bei Staphylokokken Zytoplasma, Zellmembran und Zellwand von Staphylococcus aureus. Die Vorstufen des polymeren Peptidoglykans in der Zellwand werden im Zytoplasma synthetisiert, an Lipidcarrier gebunden, in der Zytoplasmamembran verankert und dann zum weiteren Aufbau der Zellwandschichten benutzt. Das endständige Dipeptid aus zwei Aminosäuren D-Alanin (D-Ala-D-Ala) ist die Erkennungsstruktur für die Enzyme der Zellwandsynthese, wie zum Beispiel die Transpeptidase (= PBP2a). Aus [3].

Mehrere Resistenzmechanismen sind möglich:

1. Stämme, die ihre Resistenz durch Penicillinase erreichen, zerstören das Penicillin bereits außerhalb der Zelle. Penicillinase-feste Penicilline, wie z. B. Methicillin oder Flucloxacillin, sind dann noch wirksam.

2. Methicillin-resistente Stämme bilden das PBP2a - ein modifiziertes PBP (Penicillin-bindendes Protein), an das $\beta$-Laktamantibiotika nicht binden können und dadurch unwirksam sind.

3. Ceftarolin bindet an PBP2a und ist das bisher einzige therapeutisch verfügbare $\beta$-Laktamantibiotikum, das gegen MRSA wirksam ist.

\section{Einleitung}

$\nabla$

Das penicillinasefeste Penicillin Methicillin wurde vor mehr als 50 Jahren zur Behandlung von Infektionen durch Penicillinasebildende Staphylokokken entwickelt. Es war als Therapeutikum nie bedeutsam, denn bereits rasch nach der Einführung wurden resistente Staphylokokken entdeckt [1]. Einen großen Bekanntheitsgrad erlangte die Substanz trotzdem: Sie wird als Referenzsubstanz benutzt, um Staphylokokken zu charakterisieren, die nicht durch Penicillinasebildung, sondern andere Mechanismen resistent geworden sind (MRSA = Methicillin-resistente Staphylococcus aureus).

Seit dem ersten Auftreten von MRSA-Stämmen haben sich diese Erreger weltweit besonders im stationären, aber auch zunehmend im ambulanten Bereich verbreitet. Während in Europa die nosokomial entstandenen MRSA-Infektionen weiterhin dominieren, werden in den USA in einigen Regionen MRSA-Infektionen häufiger im ambulanten Bereich als im Krankenhaus beobachtet. Invasive MRSA-Infektionen gehen einher mit einer vermehrten Letalität, sie verursachen einen relativ langen Krankenhausaufenthalt und signifikant erhöhte Kosten [2]. Ursächlich für die Multiresistenz der MRSA gegen Penicilline, Cephalosporine und Carbapeneme ist eine Änderung der Penicillin-bindenden Proteine $(\mathrm{PBP})$ in diesen Bakterien. Resistente Erreger produzieren PBP2a, ein modifiziertes Protein, mit dem keines der bisher üblichen $\beta$-Laktamantibiotika interagiert. Mit Ceftarolin-Fosamil und Ceftobiprol wurden jedoch in den vergangenen Jahren erstmals $\beta$-Laktamantibiotika entwickelt, die auch bei diesen Erregern therapeutisch einsetzbar sind ( Abb.1) [3]. Sie stellen damit Alternativen zu den klassischen Substanzen wie Vancomycin, Teicoplanin oder Linezolid dar. In dieser Übersicht erfolgt eine aktuelle Bestandsaufnahme zum Stellenwert von älteren oder neueren Antibiotika in der Therapie von MRSA-Infektionen. Dabei soll insbesondere ihre mögliche Verwendung bei Infektionen der tiefen Atemwege betrachtet werden ( Tab. 1$)$.

\begin{tabular}{|c|c|c|c|c|}
\hline Arzneistoff & Handelsname & Gruppe & Tagesdosis & Indikation \\
\hline Vancomycin & diverse Generika & Glycopeptid & $\begin{array}{l}2 \times 1 \mathrm{~g} \\
\text { i.v. }\end{array}$ & CAP, HAP 1 \\
\hline Teicoplanin & Targocid & Glycopeptid & $\begin{array}{l}1 \times 400 \mathrm{mg}^{2} \\
\text { i.v. }\end{array}$ & CAP, HAP \\
\hline Telavancin & Vibativ $^{3}$ & Lipoglycopeptid & $\begin{array}{l}1 \times 10 \mathrm{mg} / \mathrm{kg} \\
\text { i.v. }\end{array}$ & HAP \\
\hline Linezolid & Zyvoxid & Oxazolidinon & $\begin{array}{l}2 \times 600 \mathrm{mg} \\
\text { i.v./p.o. }\end{array}$ & CAP, HAP \\
\hline Ceftarolin & Zinforo & Cephalosporin & $\begin{array}{l}3 \times 600 \mathrm{mg} \\
\text { i.v. }\end{array}$ & CAP \\
\hline Ceftobiprol & nicht im Handel ${ }^{4}$ & Cephalosporin & $\begin{array}{l}2-3 \times 500 \mathrm{mg} \\
\text { i.v. }\end{array}$ & $\begin{array}{l}\text { (CAP, HAP klinisch geprüft, } \\
\text { siehe Text) }\end{array}$ \\
\hline Daptomycin & Cubicin & Lipopeptid & $1 \times 4-6 \mathrm{mg} / \mathrm{kg}$ i.v. ${ }^{5}$ & $\begin{array}{l}\text { nicht indiziert bei pulmonalen } \\
\text { Infektionen }\end{array}$ \\
\hline Tigecyclin & Tygacil & Glycylcycline & $\begin{array}{l}1 \times 50 \mathrm{mg} / \mathrm{kg}^{6} \\
\text { i. v. }\end{array}$ & $\begin{array}{l}\text { nicht indiziert bei pulmonalen } \\
\text { Infektionen }\end{array}$ \\
\hline
\end{tabular}

Tab. 1 Antibiotika mit Aktivität gegen Methicillin-resistente S. aureus (MRSA).

${ }^{1}$ Laut Fachinfo ist Vancomycin bei „Pneumonie“ ohne weitere Angabe indiziert.

2 Initial werden zwei Dosen im Abstand von 12 Stunden verabreicht.

${ }^{3}$ Zurzeit in Deutschland nicht im Handel. Das Arzneimittel VIBATIV soll im Laufe des Jahres 2014 in den Handel kommen.

${ }^{4}$ Nicht im Handel. Ceftobiprol wurde von 2008 bis 2010 in der Schweiz und in Kanada unter dem Namen ZEFTERA bzw. ZEVTERA angeboten.

${ }^{5}$ Niedrige Dosis $(4 \mathrm{mg} / \mathrm{kg}$ ) nur bei komplizierten Haut- und Weichteilinfektionen ohne Bakteriämie.

${ }^{6}$ Initial wird eine Dosis von $100 \mathrm{mg}$ verabreicht. 


\begin{tabular}{|c|c|c|c|c|c|}
\hline Erreger & Vancomycin & Telavancin & Linezolid & Ceftarolin & Daptomycin \\
\hline $\begin{array}{l}\text { Staphylococcus aureus } \\
\text { (Methicillin-sensibel) }\end{array}$ & 1 & 0,5 & 2 & 0,5 & 0,5 \\
\hline $\begin{array}{l}\text { Staphylococcus aureus } \\
\text { (Methicillin-resistent) }\end{array}$ & 1 & 0,25 & 2 & 2 & 0,5 \\
\hline $\begin{array}{l}\text { Staphylococcus aureus } \\
(\text { VISA })^{1}\end{array}$ & 8 & 1 & 2 & 2 & 4 \\
\hline $\begin{array}{l}\text { Staphylococcus aureus } \\
\text { (Koagulase-negativ) }\end{array}$ & 2 & 0,5 & 1 & 0,5 & 1 \\
\hline Enterococcus faecalis ${ }^{2}$ & 2 & 1 & 2 & 8 & 1 \\
\hline Enterococcus faecium² & 1 & 0,25 & 2 & $>32$ & 4 \\
\hline $\begin{array}{l}\text { Enterokokken } \\
\text { (Van A-Resistenz) }\end{array}$ & 512 & 8 & 2 & $>32$ & 4 \\
\hline $\begin{array}{l}\text { Enterokokken } \\
\text { (Van B-Resistenz) }\end{array}$ & 512 & 2 & 2 & - & 4 \\
\hline Streptococcus pyogenes & 0,5 & 0,06 & 1 & 0,008 & 0,06 \\
\hline Streptococcus pneumoniae & 0,5 & 0,03 & 1 & 0,03 & $(0,06)$ \\
\hline Clostridium difficile & 1 & 0,25 & 8 & 4 & 2 \\
\hline
\end{tabular}

Tab. 2 In vitro-Wirkung (MHK90Werte, $\mathrm{mg} / \mathrm{l})$ einiger Antibiotika mit MRSA-Aktivität.

mod. nach [14]

${ }^{1}$ VISA = Vancomycin-intermediate $S$. aureus

2 Vancomycin-empfindliche Stämme. Die Häufigkeit Vancomycin-resistenter Enterokokken hat in Deutschland in den vergangenen Jahren deutlich zugenommen.

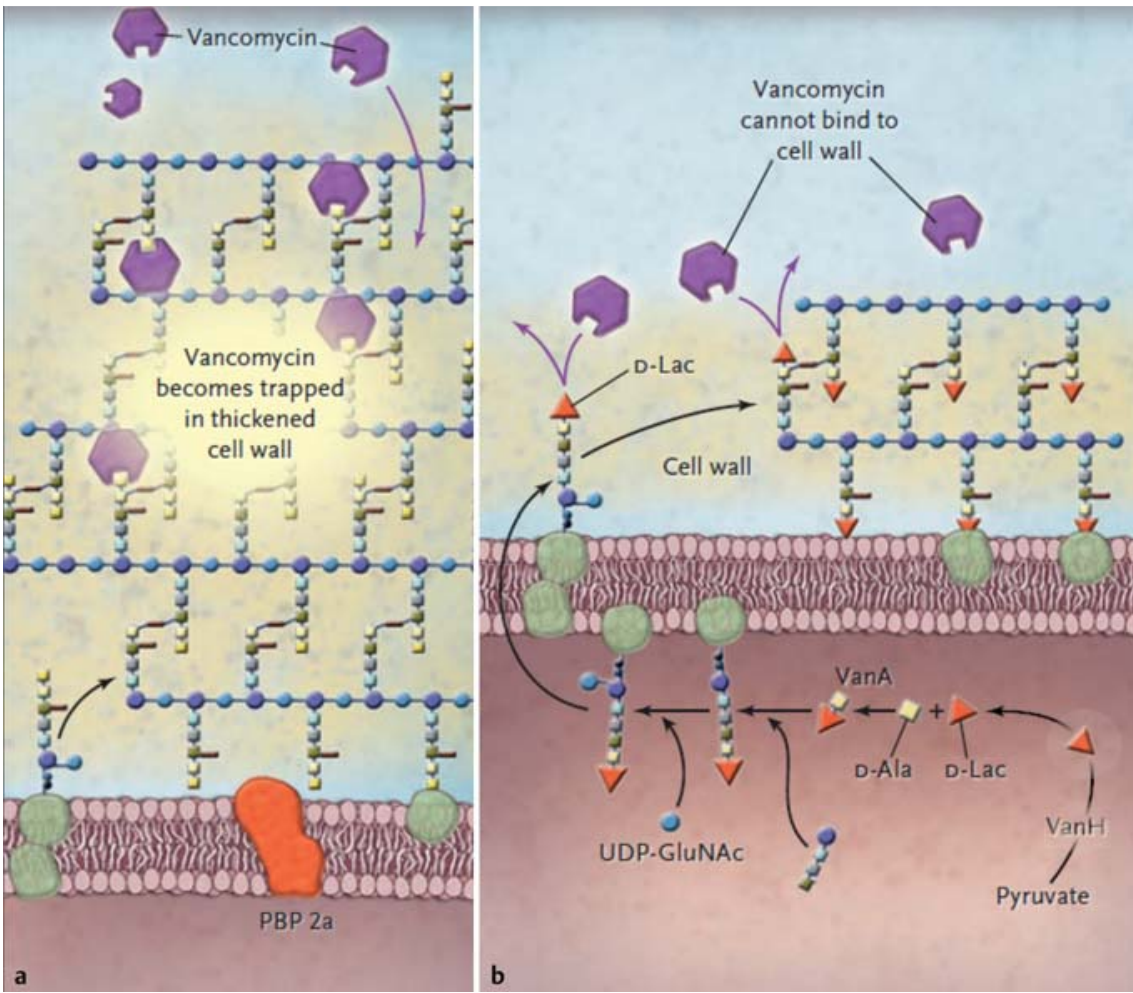

Abb. 2 Vancomycin-Resistenz bei Staphylokokken. a Vancomycin erreicht die Vorstufen des Peptidoglykans nicht, weil die Zellwand verdickt ist und die Glykopeptid-Antibiotika nicht bis zu der Zielstruktur, dem D-Alanin-D-Alanin, vordringen können. Dies ist die Ursache für eine relativ schwach ausgeprägte Resistenz. Die Stämme werden als VISA (Vancomycin-intermediär resistente S. aureus) oder GISA (Glycopeptid-intermediär resistente S. aureus) bezeichnet. b Vorstufen des Peptidoglykans sind in den resistenten Stämmen so verändert, dass Vancomycin nicht mehr binden kann. Dies wird erreicht durch Umwandlung des endständigen D-Alanins in D-Laktat (VanA, VanB, VanD Stämme) oder D-Serin (VanC, VanE, VanL). Diese Stämme sind hochresistent (VRSA, Vancomycin-resistente $S$. aureus). Die Gene, die diese Resistenz vermitteln (vanA), wurden primär in Enterokokken nachgewiesen. Sie sind bei S. aureus zur Zeit noch selten. Aus [3].

\section{Vancomycin, Teicoplanin}

$\nabla$

Etwa zur gleichen Zeit wie Methicillin kam auch Vancomycin auf den Markt, seine Bedeutung stieg allerdings erst Anfang der 1980er Jahre, als es zunehmend bei MRSA-Infektionen und bei der Clostridium difficile-assoziierten Kolitis Anwendung fand [4]. Nach wie vor stellt es das Standardtherapeutikum bei MRSA-Infektionen dar. Mit Teicoplanin gibt es seit 1989 ein weiteres Glycopeptid, das sich vor allem in den pharmakokinetischen Eigenschaften von Vancomycin unterscheidet.

Glykopeptid-Antibiotika hemmen die Zellwandsynthese grampositiver Bakterien, indem sie die Peptidoglykansynthese durch Bindung an das Substrat hemmen. Damit unterscheiden sie sich von den $\beta$-Laktamantibiotika, welche die Transpeptidase und andere Enzyme der Zellwandsynthese hemmen. In vitro zeigen Vancomycin und Teicoplanin nur geringe Aktivitätsunterschiede. Ihr Spektrum ist auf grampositive Erreger begrenzt ( Tab.2). Zwischen Vancomycin und Teicoplanin besteht eine partielle Kreuzresistenz. Einige Vancomycin-resistente Enterokokken sind Teicoplanin-empfindlich (VanB-Phänotyp). Besorgniserregend ist die seltene, aber in einigen Ländern zunehmende Resistenz bei Staphylokokken ( Abb.2) [3]. Auch bei Enterococcus faecium werden zunehmend Glykopeptid-resistente Stämme isoliert. Ein dramatischer Anstieg der Vancomycin-resistenten Enterokokken in Deutschland wurde zwischen 2007 und 2012 festge- 
Tab.3 Unerwünschte Wirkungen einiger Antibiotika mit MRSA-Aktivität

\begin{tabular}{|c|c|}
\hline Arzneistoff & Unerwünschte Wirkungen, Toxizitätsrisiken \\
\hline Vancomycin & $\begin{array}{l}\text { Ototoxizität, Nephrotoxizität (vor allem bei höheren } \\
\text { Dosen), red man syndrome (bei zu rascher Infusion) }\end{array}$ \\
\hline Teicoplanin & $\begin{array}{l}\text { Ototoxizität, Nephrotoxizität (vor allem bei höheren } \\
\text { Dosen) }\end{array}$ \\
\hline Telavancin & $\begin{array}{l}\text { Störungen des Magendarmtrakts (Dysgeusie), geringe } \\
\text { QT-Verlängerung; Nephrotoxizität }\end{array}$ \\
\hline Linezolid & $\begin{array}{l}\text { Thrombozytopenie, Anämie, Neuropathie (abhängig } \\
\text { von der Therapiedauer), Laktatazidose, Serotonin-Syn- } \\
\text { drom }\end{array}$ \\
\hline Ceftarolin & Störungen des Magendarmtrakts, Exantheme \\
\hline Daptomycin & $\begin{array}{l}\text { CPK-Anstieg, Myopathie, Rhabdomyolyse (selten), } \\
\text { periphere Neuropathie, eosinophile Pneumonie (selten) }\end{array}$ \\
\hline Tigecyclin & $\begin{array}{l}\text { Störungen des Magendarmtrakts (häufig Übelkeit, } \\
\text { Erbrechen) }\end{array}$ \\
\hline
\end{tabular}

mod. nach [42]

stellt. Mittlerweile sind mehr als 10\% der Stämme, die auf intensivmedizinischen Stationen isoliert werden, resistent [5].

Nach oraler Gabe werden Vancomycin und Teicoplanin nicht resorbiert. Dies wird bei der Behandlung der „pseudomembranösen Kolitis“ durch Clostridium difficile ausgenutzt, um nach oraler Gabe hohe Konzentrationen im Darm zu erreichen. Nach intravenöser Infusion ist die Plasmahalbwertzeit von Teicoplanin etwa zehnfach länger als die von Vancomycin, wodurch eine einmal tägliche Dosierung möglich wird. Beide Antibiotika werden kaum metabolisiert und sind nicht hämodialysierbar. Die Gewebegängigkeit der Glykopeptide ist nicht optimal: Im epithelialen Flüssigkeitsfilm (ELF) können etwa 50\% der Plasmakonzentrationen erwartet werden, in Alveolarmakrophagen wurden Konzentrationen von ca. $45 \mathrm{mg} / \mathrm{l}$ gemessen [6].

Überempfindlichkeitsreaktionen mit Fieber, Urtikaria und Exanthemen können bei einer Therapie mit Glykopeptiden auftreten. Ein Blutdruckabfall mit Erythem am Oberkörper („Red-Man-Syndrom“) kann bei zu schneller Infusion von Vancomycin durch Histaminfreisetzung verursacht werden, dieses Phänomen tritt bei Teicoplanin nicht auf. Bei Behandlung mit Vancomycin oder Teicoplanin kann eine Verschlechterung des Hörvermögens auftreten. Schmerzen und Phlebitis an der Infusionsstelle sind möglich. Hohe Dosen von Glycopeptiden können ein Nierenversagen verursachen; das Risiko einer reversiblen Nephrotoxizität ist nach Vancomycin höher als nach Teicoplanin [7]. Vorsicht ist vor allem bei gleichzeitiger Gabe von anderen potenziell nephrotoxischen Medikamenten geboten ( $\bullet$ Tab.3).

Bei etwa einem Drittel der Patienten mit einer hohen Dosierung von $4 \mathrm{~g}$ Vancomycin und mehr pro Tag ließ sich eine nephrotoxische Reaktion nachweisen im Vergleich zu etwa $11 \%$ der Patienten mit einer niedrigen bzw. normalen Dosis. Eine primär eingeschränkte Nierenfunktion und ein hohes Körpergewicht führen vermehrt zu nephrotoxischen Reaktionen. Der Quotient aus AUC (Fläche unter der Konzentrationszeitkurve, $24 \mathrm{~h}$ ) und der minimalen Hemmkonzentration (MHK) ist ein wesentlicher pharmakologischer Parameter für die Wirksamkeit der Glycopeptide; es sollten für diesen Quotienten Werte von 350 bis 400 für Vancomycin angestrebt werden. Damit ist eine Vancomycin-Therapie insbesondere bei MHK-Werten der MRSA von mehr als $1 \mathrm{mg} / \mathrm{l}$ problematisch [8].

Die zunehmende Zahl übergewichtiger Patienten stellt immer häufiger ein Problem dar, wenn es um die optimale Dosierung von Antibiotika geht. Bei einer Dosiswahl nach Körpergewicht bleibt bei adipösen Patienten unberücksichtigt, dass sich das Verteilungsvolumen des Antibiotikums nicht proportional mit dem Gesamtkörpergewicht erhöht [9]. Für Vancomycin wird zunächst eine Dosierung nach Gesamtgewicht empfohlen, anschließend sollte die Dosierung durch ein Drug Monitoring optimiert werden, da keine ausreichenden Erfahrungen für andere Empfehlungen vorliegen. Als Ziel wird heute eine Einhaltung von Talspiegeln zwischen 15 und $20 \mathrm{mg} / \mathrm{l}$ angesehen. Das Nephrotoxizitätsrisiko war etwa verdreifacht bei Patienten mit Talkonzentrationen von $>15 \mathrm{mg}$ Vancomycin/l Plasma im Vergleich zu den Patienten mit niedrigeren Konzentrationen [10]. Mehrere kleine Studien deuten darauf hin, dass auch eine kontinuierliche Infusion von Vancomycin über 24 Stunden anstatt der üblichen zweimal täglichen Kurzinfusion sinnvoll sein könnte. Tendenziell wurden nephrotoxische Reaktionen bei Dauerinfusion seltener beobachtet, derzeit erlaubt die Datenlage jedoch noch keine generelle Empfehlung für diese Maßnahme [11,12].

\section{Telavancin}

$\nabla$

Telavancin ist ein halbsynthetisches Derivat des Vancomycins, das als Lipoglycopeptid bezeichnet wird. Es hemmt die Zellwandsynthese der Bakterien und stört die Funktion der Zellmembran. Die in vitro-Aktivität ist hoch, auch Vancomycin-resistente Enterokokken und Staphylokokken werden erfasst ( $\bullet$ Tab.2). Nach einer zweistündigen Infusion von Telavancin in einer Dosis von $10 \mathrm{mg} / \mathrm{kg}$ wurde eine mittlere Spitzenkonzentration von $88 \mathrm{mg} / \mathrm{l}$ im Plasma gemessen, der AUC-Wert wurde mit $858 \mathrm{mg} / \mathrm{l} \times \mathrm{h}$ errechnet. Telavancin wird zu etwa $93 \%$ an Plasmaproteine gebunden, das Verteilungsvolumen liegt bei $0,11 / \mathrm{kg}$. Im epithelialen Flüssigkeitsfilm (ELF) lagen Konzentrationen von Telavancin acht Stunden nach einer Infusion bei $3,7 \mathrm{mg} / 1$. In den Alveolarmakrophagen wurden 12 und 24 Stunden nach der Verabreichung Konzentrationen von 45 und $42 \mathrm{mg} / \mathrm{l}$ gemessen (Plasmakonzentrationen: 22,9 und 7,3 mg/l). Etwa zwei Drittel einer verabreichten Dosis werden unverändert im Urin ausgeschieden, bei eingeschränkter Nierenfunktion ( $\mathrm{ClCr} 30$ bis $50 \mathrm{ml} / \mathrm{min}$ ) soll die Dosierung von 10 auf $7,5 \mathrm{mg} / \mathrm{kg}$ reduziert werden, bei deutlicherer Einschränkung ( $<30 \mathrm{ml} / \mathrm{min}$ ) wird eine Dosis von $10 \mathrm{mg} / \mathrm{kg}$ nur alle 48 Stunden verabreicht $[13,14]$.

In zwei umfangreichen klinischen Phase-3-Studien (ATTAIN, Assessment of Telavancin for Treatment of Hospital-Acquired Pneumonia) wurden mehr als 1500 Patienten mit HAP (hospital acquired pneumonia) entweder mit Telavancin $(10 \mathrm{mg} / \mathrm{kg}$ alle 24 Stunden) oder Vancomycin (1,0g alle 12 Stunden) behandelt. Bei mehr als der Hälfte der Patienten lag eine Infektion mit MRSA vor. Unter den 654 klinisch auswertbaren Fällen lag die Heilungsrate für Telavancin in diesen Doppelblindstudien bei 82,4\% und für Vancomycin bei 80,7\%. Auch Art und Häufigkeit der unerwünschten Ereignisse waren in beiden Gruppen sehr ähnlich. Die Kreatininkonzentration war bei den Patienten der Telavancin-Gruppe am Therapieende häufiger erhöht (16\% vs. $10 \%$ ).

Die Überlebensraten innerhalb von 28 Tagen nach Beginn der Behandlung waren für Patienten ohne wesentliche Einschränkung der Nierenfunktion $(\mathrm{ClCr}>50 \mathrm{ml} / \mathrm{min})$ in beiden Behandlungsgruppen sehr ähnlich, sie zeigten jedoch größere Unterschiede, wenn eine mäßig bis stark ausgeprägte Niereninsuffizienz vorlag. Bei einer Kreatinin-Clearance von $<30 \mathrm{ml} / \mathrm{min}$ überlebten in den vier Wochen nach Studienbeginn 61\% bzw. 47\% der Patienten $[15,16]$. 


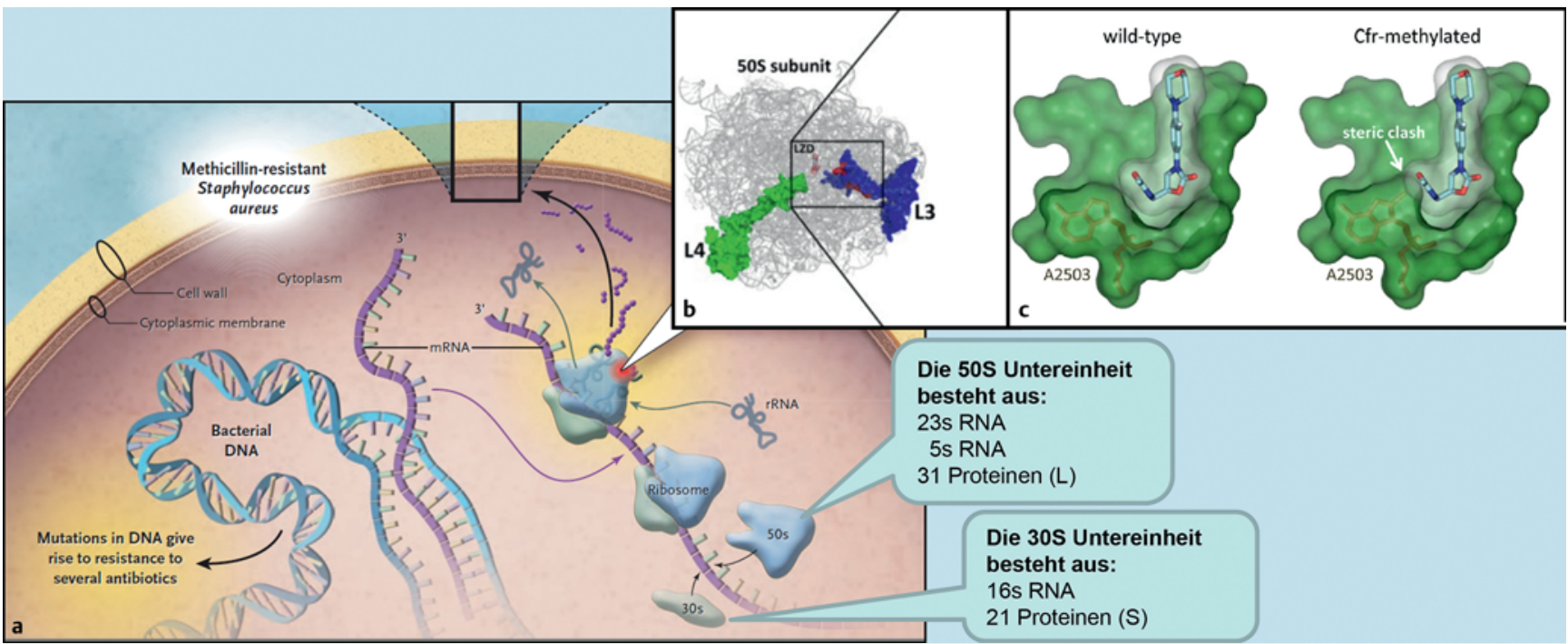

Abb. 3 Linezolid-Resistenz bei Staphylokokken durch ribosomale Veränderungen (a) Durch Veränderungen in der rRNA oder den Proteinen L3 bzw. L4 der ribosomalen 50S Untereinheit können MRSA-Stämme Resistenz gegen Linezolid erlangen. Am häufigsten sind Mutationen, die zum Austausch von Basen in der rRNA führen. Durch weitere Mutationen können die MRSA auch resistent gegen zahlreiche andere Antibiotika werden. (b) Dieser Teil der Abbildung zeigt die 50S-Untereinheit mit Veränderungen in dem L3-Protein, die in der Nähe der Bindungsstelle von Linezolid im Peptidyltransferasezentrum auftreten ( $\Delta$ Ser145/ His146Tyr und $\Delta$ Met169-Gly174). (c) Besorgniserregend ist die Plasmid-kodierte Cfr-basierte Resistenz, weil hier eine horizontale Ausbreitung befürchtet werden muss. Das cfr-Gen wurde im Jahr 2000 als Ursache für Chloramphenicol-(bzw. Florfenicol)-Resistenz in Proben von Tieren entdeckt. Es kodiert für eine Methyltransferase, welche das Adenin A2503 in der 23S rRNA in Position 8 methyliert und die Bindung eines Linezolid-(LZD)-Moleküls an das Peptidyltransferasezentrum beeinträchtigt. Diese geringe Veränderung verhindert die Bindung von fünf chemisch sehr unterschiedlichen Klassen von Antibiotika: den Phenicolen, Lincosamiden, Oxazolidinonen, Pleuromutilinen und Streptograminen [3,20,22].

In tierexperimentellen Studien wurden teratogene Effekte beobachtet, die bei Dosierungen auftraten, die der Exposition des Menschen nach Standarddosierungen entsprechen. Vor einer Behandlung von Frauen im gebärfähigen Alter sollte eine Schwangerschaft ausgeschlossen werden [17].

In den USA und Kanada ist das Antibiotikum zur Behandlung der im Krankenhaus erworbenen bakteriellen Pneumonie und beatmungsassoziierten Pneumonie zugelassen. Es soll jedoch nur angewandt werden, wenn andere Alternativen nicht in Frage kommen. In Europa wurde die Zulassung von der EMA bereits im Mai 2011 erteilt, die Markteinführung des Präparates in Europa ist im Laufe des Jahres 2014 vorgesehen.

\section{Linezolid \\ $\nabla$}

Linezolid wird bei Infektionen durch MRSA, VRE (Vancomycinresistente Enterokokken) oder andere grampositive Erreger heute weltweit eingesetzt. Die Substanz bindet an die 23S rRNA in der 50S-Untereinheit der bakteriellen Ribosomen und wirkt auf diese Weise bakteriostatisch [18]. Da es sich um das erste Arzneimittel aus dieser Wirkstoffgruppe handelt, sind Daten zur Resistenzentwicklung von besonderem Interesse. Unter den Streptokokken, einschließlich S. pneumoniae, sind bisher keine resistenten Stämme nachgewiesen worden, bei den koagulasenegativen Staphylokokken liegt die Rate aktuell bei 0,9\% und bei den Enterokokken bei $0,8 \%$. Unter etwa $4000 \mathrm{~S}$. aureus-Isolaten, die im Jahr 2012 untersucht worden sind, fanden sich nur vier Stämme, die bei $2 \mathrm{mg} / \mathrm{l}$ nicht gehemmt wurden. Sie wurden in Italien, Hongkong und Brasilien isoliert. Verschiedene Mechanismen lagen der Resistenzentwicklung zugrunde. Es handelte sich um verschiedene Veränderungen der Ribosomen, in denen entweder die 23 S rRNA oder die ribosomalen Proteine L3 und L4 in der 50S-
Untereinheit verändert waren. Bemerkenswert ist der Nachweis eines Stammes mit dem cfr-Gen in Brasilien. Cfr-positive Stämme sind seit 2000 bekannt und waren im Jahr 2008 in Spanien erstmals als Verursacher eines Ausbruchs von Infektionen bei insgesamt 15 Patienten in einem Krankenhaus in Madrid identifiziert worden [19]. Das Gen kodiert für eine Methyltransferase, welche Adenosin in Position 2503 in der 23S rRNA modifiziert und dadurch die Bindung von Linezolid und weiteren Antibiotika aus anderen Klassen an das Peptidyltransferasezentrum beeinträchtigt ( Abb.3) [20]. Da es Plasmid-kodiert ist, muss eine horizontale Verbreitung befürchtet werden [21]. Ein neueres Oxazolidinon, das derzeit klinisch geprüft wird, ist Tedizolid (=Sivextro ${ }^{\circledR}$ ). Es besitzt eine höhere in vitro-Aktivität als Linezolid und scheint auch gegen Staphylokokken wirksam zu sein, die das cfr-Gen besitzen [22,23].

Linezolid wird nach oraler Gabe gut resorbiert und kann daher in der Sequenz-Therapie eingesetzt werden. Diese Möglichkeit eines Wechsels von intravenöser auf orale Behandlung kann die Aufenthaltsdauer im Krankenhaus im Vergleich zur VancomycinTherapie verkürzen. Nach mehrfacher oraler Gabe von $600 \mathrm{mg}$ Linezolid im Abstand von 12 Stunden wurden Spitzenkonzentrationen von $21 \mathrm{mg} / \mathrm{l}$ und Talkonzentrationen $6 \mathrm{mg} / \mathrm{l}$ erreicht. Die Plasmaproteinbindung von Linezolid ist mit ca. $30 \%$ niedrig, das Verteilungsvolumen wurde mit etwa 0,6 bis $0,71 / \mathrm{kg}$ berechnet. Linezolid wird überwiegend zu zwei Hauptmetaboliten abgebaut, die dann neben der unveränderten Substanz im Urin eliminiert werden. Die Eliminationshalbwertzeit liegt bei 5 bis 7 Stunden [24].

Die Gewebegängigkeit von Linezolid wurde bei erwachsenen Patienten untersucht. Die mittlere Konzentration des Linezolids im Serum betrug $13,4 \mathrm{mg} / \mathrm{l}$, die entsprechenden pulmonalen Konzentrationen lagen in den Makrophagen bei $8,1 \mathrm{mg} / \mathrm{l}$, in der epithelialen Oberflächenflüssigkeit der Alveolen bei $25,1 \mathrm{mg} / \mathrm{l}$ und 
in Biopsieproben der bronchialen Mukosa bei 10,7 mg/kg. Diese Resultate bestätigen die relativ gute Gewebegängigkeit des Linezolids [25].

Bei den unerwünschten Wirkungen stehen gastrointestinale Störungen und leichte ZNS-Symptome im Vordergrund ( Tab.3). Bei längerer Behandlungsdauer ( $>2$ Wochen) kann es zu einer Thrombozytopenie kommen, die offenbar bei Patienten mit Niereninsuffizienz relativ häufig ist [26]. Eine wöchentliche Blutbildkontrolle ist bei allen Patienten unabhängig von den Ausgangswerten des Blutbildes angezeigt. Linezolid hemmt die mitochondriale Proteinsynthese. Als Folge dieser Hemmung können Nebenwirkungen wie z.B. Laktatazidose, Anämie und optische und periphere Neuropathie auftreten. Derartige Erscheinungen sind häufiger, wenn das Arzneimittel länger als 28 Tage angewendet wird. Nach längerer Behandlungsdauer kam es in Einzelfällen zu einem irreversiblen Sehverlust. Linezolid ist ein reversibler, nichtselektiver Hemmstoff der Monoaminooxidase (MAO). Bei gleichzeitiger Gabe von Linezolid und serotonergen Wirkstoffen, einschließlich Antidepressiva wie selektive Serotonin-Wiederaufnahme-Hemmer (SSRIs), wurden Fälle eines SerotoninSyndroms berichtet. Die gleichzeitige Gabe von Linezolid und einem serotonergen Wirkstoff ist daher kontraindiziert. Es sollte ebenfalls nicht eingesetzt werden, wenn eines der folgenden Arzneimittel angewandt wird: Serotonin-Agonisten („Triptane“), Sympathomimetika (einschließlich adrenerger Bronchodilatatoren, Pseudoephedrin oder Phenylpropanolamin), vasopressorische Arzneistoffe (z.B. Adrenalin, Noradrenalin), dopaminerge Mittel (z.B. Dopamin, Dobutamin), Pethidin oder Buspiron. Im Rahmen der präklinischen, toxikologischen Prüfung kam es bei Ratten zu einer Abnahme der Fertilität und Reproduktionsleistung bei humantherapeutisch relevanten Expositionen. Bei geschlechtsreifen Tieren waren diese Wirkungen reversibel, bei Jungtieren jedoch nicht. Die Bedeutung dieser Befunde für den Menschen ist unklar.

Die Vergleichsstudien mit Vancomycin zeigen entweder eine therapeutische Gleichwertigkeit der beiden Stoffe oder eine Überlegenheit des Linezolids. In den internationalen Leitlinien werden bei HAP oder VAP heute entweder Vancomycin oder Linezolid empfohlen. In der neuesten Doppelblindstudie wurden Vancomycin und Linezolid bei Patienten mit MRSA-Pneumonie verglichen [27]. Die Mehrzahl der MRSA-Nachweise erfolgte über Tracheal-Aspirate $(43,4 \%)$ und bronchoalveolärer Lavage $(31,3 \%)$. Blutkulturen waren zu 6,6\% positiv. Linezolid (600 mg) oder Vancomycin $(15 \mathrm{mg} / \mathrm{kg}$ ) wurden alle 12 Stunden intravenös gegeben. Die Vancomycin-Dosis wurde anhand der gemessenen Talkonzentrationen optimiert. Die Patienten erhielten zusätzlich ein Antibiotikum mit Aktivität gegen gramnegative Erreger. Die klinischen Ergebnisse am Ende der Studie zeigten mit 58\% (95/ $165)$ erfolgreichen Behandlungen in der Linezolidgruppe einen signifikant besseren Effekt im Vergleich zu 47\% (81/174) in der Vancomycingruppe $(\mathrm{p}=0,04)$. Insgesamt traten bei $18 \%$ der Patienten unter Vancomycin nephrotoxische Wirkungen auf $(8,4 \%$ unter Linezolid), die Rate von hämatologischen Veränderungen, wie Anämie oder Thrombozytopenie war in beiden Gruppen sehr ähnlich. Die Gesamtletalität zum Tag 60 der Studie (Linezolid $15,7 \%$, Vancomycin 17,0\%) unterschied sich nicht signifikant zwischen den beiden Substanzen [27]. Linezolid ist sicher eine interessante Alternative zu den Glykopeptiden, insbesondere bei Patienten mit eingeschränkter Nierenfunktion, bei mäßig Vancomycin empfindlichen MRSA-Stämmen und auch bei Patienten, die zusätzlich potenziell nephrotoxische Substanzen erhalten [28].

\section{Ceftarolin}

\section{$\nabla$}

Ceftarolin-Fosamil ist das erste in Deutschland verfügbare $\beta$-Laktamantibiotikum, welches auch das PBP2a bei MRSA inaktiviert und eine gute antibakterielle Aktivität gegen diese und andere grampositive Erreger besitzt ( $\bullet$ Abb. 1, $\square$ Tab.2). Auch bei einigen gramnegativen Bakterien, wie Haemophilus influenzae, Moraxella catarrhalis oder Neisseria meningitidis, wurde eine hohe Aktivität mit $\mathrm{MHK}_{90}$-Werten unter $0,5 \mathrm{mg} / \mathrm{l}$ ermittelt. Ceftarolin wird durch einige $\beta$-Laktamasen, wie zum Beispiel die ESBL (extended spectrum $\beta$-lactamases) und AmpC-Enzyme, aber auch durch die TEM- und SHV-Penicillinasen zerstört. Die mangelnde $\beta$-Laktamasestabilität ist sehr ungewöhnlich für ein Cephalosporin mit Oxyimino-Struktur. Durch Kombination der Substanz mit einem $\beta$-Laktamaseinhibitor kann diesem Nachteil begegnet werden. In klinischer Prüfung befindet sich die Kombination mit Avibactam, einem Inhibitor, der nicht zu den $\beta$-Laktamverbindungen gehört [29].

Das Cephalosporin wird zweimal täglich in einer Dosierung von $600 \mathrm{mg}$ intravenös über eine Stunde verabreicht, die maximalen Plasmakonzentrationen liegen bei etwa $21 \mathrm{mg} / \mathrm{l}$, der AUC-Wert wurde mit $56 \pm 9 \mathrm{mg} / \mathrm{l} \times \mathrm{h}$ berechnet. Ceftarolin wird nur in geringem Ausmaß an Plasmaproteine gebunden ( $<20 \%)$, das Verteilungsvolumen entspricht mit ca. 20 Litern etwa dem Extrazellulärraum. Das Antibiotikum wird überwiegend unverändert renal mit einer Halbwertzeit von 2,6 Stunden eliminiert. Bei Patienten mit eingeschränkter Nierenfunktion $\left(\mathrm{Cl}_{\mathrm{Cr}}\right.$ zwischen 30 und $50 \mathrm{ml} /$ min) wird empfohlen, eine reduzierte Dosis von zweimal täglich $400 \mathrm{mg}$ zu verabreichen.

Ceftarolin ist zur Behandlung von komplizierten Haut- und Weichgewebsinfektionen zugelassen sowie zur Therapie der ambulant erworbenen Pneumonie. Es erwies sich als mindestens gleichwertig wirksam wie die Standardantibiotika. Bei fast $80 \%$ der Patienten mit Wundinfektionen, Abszessen oder anderen Weichgewebsinfektionen waren Staphylokokken die ursächlichen Erreger. Auch bei MRSA-bedingten Infektionen konnten keine Unterschiede im Therapieergebnis zu Vancomycin ermittelt werden [30].

Der Vergleich bei ambulant erworbener Pneumonie erfolgte gegen Ceftriaxon. In zwei Doppelblindstudien wurden die Wirksamkeit und Verträglichkeit von Ceftarolin $(2 \times$ tgl. $600 \mathrm{mg}$ i.v. $)$ im Vergleich zu Ceftriaxon in einer Dosierung von einmal täglich 1,0 g i.v. untersucht $[31,32]$. Nach dem Schweregrad der Pneumonie gehörten alle Patienten zu den Gruppen PORT III und IV. Ein Erreger konnte bei 27\% der Patienten isoliert werden, dominierend war S. pneumoniae. Die Heilungsraten nach klinischen Kriterien lagen bei $84 \%$ (Ceftarolin) und bei 78\% (Ceftriaxon). In den Zulassungsstudien erwies sich Ceftarolin als sehr gut verträglich. Zu den unerwünschten Symptomen, die während der Behandlung auftraten, zählten Diarrhö, Kopfschmerzen, Übelkeit, und Juckreiz.

Die Studien geben keinen Hinweis auf eine etwaige Eignung des Cephalosporins bei MRSA-verursachten Pneumonien, denn Patienten mit Verdacht oder Nachweis einer Infektion durch MRSA, ebenso wie mit atypischen oder nosokomial erworbenen Erregern, waren in diesen klinischen Studien ausgeschlossen. Gezielte Untersuchungen bei nosokomialer Pneumonie mit MRSA liegen nicht vor. Unter www.clinicaltrials.gov werden insgesamt 31 klinische Studien mit Ceftarolin aufgeführt, allerdings keine doppelblind-randomisierte Studie zur möglichen Anwendung bei nosokomialen MRSA-Pneumonien. In einer retrospektiven Auswertung wird über 92 Patienten mit Pneumonie berichtet, 
von denen 68 an einer nosokomialen und/oder MRSA-Pneumonie erkrankt waren und die damit off label behandelt wurden. Einige Patienten erhielten $600 \mathrm{mg}$ Ceftarolin dreimal anstatt zweimal täglich, insgesamt wurden $86 \%$ der Patienten unter Berücksichtigung klinischer Kriterien erfolgreich behandelt. Wegen des retrospektiven Designs dieser Beobachtungsstudie können allerdings keine weitreichenden Empfehlungen daraus abgeleitet werden [33].

\section{Ceftobiprol}

$\nabla$

Ceftobiprol ist ein weiteres Cephalosporin, das in vitro gegen MRSA aktiv ist. Die $\mathrm{MHK}_{90}$-Werte liegen bei $2 \mathrm{mg} / \mathrm{l}$. Die Zulassungsstudien zeigten eine Nichtunterlegenheit im Vergleich zu Vancomycin bei komplizierten Haut- und Weichgewebsinfektionen. In Doppelblindstudien wurde es auch bei ambulant (CAP) und bei nosokomial erworbener Pneumonie (HAP) in einer Dosierung von $3 \times \operatorname{tgl}$. $500 \mathrm{mg}$ geprüft. In der HAP-Studie diente die Kombination aus Ceftazidim $(3 \times$ tgl. 2 g) und Linezolid $(2 \times$ tgl. $600 \mathrm{mg}$ ) als Vergleich. Obwohl die Gruppe der erfolgreich Behandelten in beiden Studienarmen bei etwa 50\% lag (intentionto-treat subjects), wurden bei den beatmeten Patienten (ventilator-associated pneumonia, VAP) mit Ceftobiprol weniger Patienten erfolgreich behandelt als in der Gruppe mit der Kombinationstherapie (23,5\% [24/102] vs. 36,2\% [38/105]). Eine Subgruppenanalyse zeigte, dass in der Ceftobiprolgruppe deutlich mehr Männer mit schweren Traumata behandelt worden waren als in der Vergleichsgruppe (15/24 vs. 3/17). Da bei kritisch kranken VAP-Patienten oft eine erhöhte Clearance und ein erhöhtes Verteilungsvolumen bestehen, kann vermutet werden, dass die Behandlung mit Ceftobiprol aufgrund der relativ niedrigen Dosierung und unterschiedlicher Gruppenverteilung der Patienten nicht überzeugend war. Bei $\beta$-Laktamantibiotika stellt die Zeitdauer der Konzentration oberhalb der minimalen Hemmkonzentration des Erregers den wichtigsten pharmakologischen Parameter für einen Therapieerfolg dar. Ceftobiprol war für kurze Zeit in der Schweiz, Kanada und einigen anderen Ländern im Handel, es steht heute jedoch nicht mehr zur Therapie zur Verfügung [34].

\section{Daptomycin (nicht zugelassen bei Pneumonie) \\ $\nabla$}

Daptomycin ist ein zyklisches Lipopeptid. Struktur und Wirkmechanismus unterscheiden die Substanz von anderen therapeutisch genutzten Antibiotika. Die bakterizid wirksame Substanz bindet an die Zytoplasmamembran durch eine Calcium-abhängige Insertion und bildet Ionenkanäle. Durch einen Austritt von Kalium kommt es zur Depolarisation der Zytoplasmamembran. Daptomycin wird einmal täglich intravenös appliziert und hauptsächlich renal eliminiert. Das Verteilungsvolumen liegt bei $0,1 \mathrm{l} / \mathrm{kg}$, die Halbwertzeit beträgt 8 bis 9 Stunden.

Daptomycin ist für die Behandlung komplizierter Haut- und Weichgewebsinfektionen sowie bei Bakteriämie und Rechtsherz-Endokarditis durch S. aureus seit 2006 zugelassen. Die klinischen Erfahrungen mit dem Antibiotikum unter Alltagsbedingungen werden in einem Register dokumentiert, das vom Hersteller des Präparates initiiert wurde. Demnach hat sich das Antibiotikum bei Infektionen durch $S$. aureus und andere grampositive Erreger bewährt. Unerwünschte Ereignisse wurden bei etwa $17 \%$ der Patienten registriert. Ein Anstieg der Kreatinphospho- kinase (CPK) und myotoxische Komplikationen traten bei relativer Überdosierung auf ( Tab.3). Die CPK muss bei Daptomycinbehandelten Patienten mindestens einmal pro Woche kontrolliert werden und das Dosierungsintervall muss bei eingeschränkter Nierenfunktion verlängert werden $[35,36]$.

Trotz guter in vitro-Aktivität gegen S. pneumoniae $\left(\mathrm{MHK}_{90}: 0,06\right.$ $\mathrm{mg} / \mathrm{l}$ ) waren die Therapieresultate in einer Phase 3 Studie bei Patienten mit ambulant erworbener Pneumonie nicht zufriedenstellend. Im direkten Vergleich zwischen Ceftriaxon $(1 \times \operatorname{tgl} .2,0 \mathrm{~g})$ und Daptomycin $(1 \times \operatorname{tgl} .4 \mathrm{mg} / \mathrm{kg})$ wurde die klinische Wirksamkeit mit 79\% für Daptomycin und 87\% für Ceftriaxon festgestellt. Als Ursache für die relativ schlechte Wirksamkeit wurde eine Inaktivierung des Antibiotikums durch Surfactant ermittelt. Dies erklärt, warum nur bei Infektionen der Lunge die Therapieresultate nicht mit der in vitro-Aktivität korrelieren. Gezielte in vitroUntersuchungen zeigten, dass in Gegenwart von $10 \%$ Surfactant die antibakterielle Aktivität um mehr als das 100-fache reduziert ist. Auch Experimente mit Mäusen zeigen, dass bei einer broncho-alveolären Pneumonie durch Streptokokken oder Staphylokokken Daptomycin praktisch unwirksam war, während mit Ceftriaxon eine deutliche Keimreduktion von etwa fünf Größenordnungen erzielt werden konnte. Interessanterweise war Daptomycin jedoch etwa gleich wirksam wie Ciprofloxacin bei Mäusen mit inhalativer Anthrax-Exposition [37].

Aktuelle Studien zeigen bei kritisch kranken, septischen Patienten eine sehr hohe Daptomycin-Clearance und niedrige SerumKonzentrationen, was zu einem mangelhaften Therapieerfolg führen kann. Die übliche Dosierung von Daptomycin wird mit $6 \mathrm{mg} / \mathrm{kg}$ Körpergewicht bei normaler Nierenfunktion angegeben. In einer aktuellen Studie wurde festgestellt, dass in einer Gruppe von 50 Patienten mit Staphylokokken-Infektionen etwa jeder Vierte eine stark erhöhte Daptomycin-Clearance (1,811/h vs. $0,751 \mathrm{l} / \mathrm{h}$ ) und dementsprechend niedrigere AUC-Werte aufwies (273 vs. $564 \mathrm{mg} / \mathrm{L} \times \mathrm{h})$. Alle Patienten mit erhöhter Clearance litten an einer Sepsis und/oder bakteriellen Endokarditis, und die Letalität war in dieser Patientengruppe deutlich erhöht. Die Autoren schlagen vor, generell eine höhere Dosis von $750 \mathrm{mg}$ Daptomycin täglich bei derartig kritisch kranken, septischen Patienten anzuwenden bzw. ein therapeutisches Drug Monitoring durchzuführen [38].

\section{Tigecyclin (nicht zugelassen bei Pneumonie) \\ $\nabla$}

Tigecyclin ist ein Derivat des Minocyclins, es wird als Glycylcyclin bezeichnet. Ähnlich wie die Tetracycline hemmt auch Tigecyclin die bakterielle Proteinbiosynthese durch Anlagerung an die 30SUntereinheit der Ribosomen und wirkt gegen ein breites Spektrum von grampositiven und gramnegativen Erregern. Glycylcycline binden mit höherer Affinität ans Ribosom und zeigen auch Aktivität gegen Tetracyclin-resistente Bakterien. Zum Spektrum gehören auch MRSA mit einem $\mathrm{MHK}_{90}$-Wert von 0,25 bis $0,5 \mathrm{mg} / \mathrm{l}$ und Vancomycin-resistente E. faecalis-Stämme (VRE). Tigecyclin wird als Infusion verabreicht; die empfohlene Dosierung beträgt $50 \mathrm{mg}$ alle 12 Stunden, wobei initial einmalig die doppelte Dosis gegeben wird. Ein „steady state“ wird nach drei bis vier Tagen erreicht. Die Spitzenkonzentrationen im Plasma liegen bei $0,6 \mathrm{mg} / \mathrm{l}$, die AUC-Werte wurden mit etwa $3 \mathrm{mg} / \mathrm{l} \times \mathrm{h}$ bzw. $4,7 \mathrm{mg} / \mathrm{l} \times \mathrm{h}$ errechnet $[39,40]$. Die Gewebegängigkeit von Tigecyclin ist gut, das scheinbare Verteilungsvolumen liegt bei 7 bis $91 / \mathrm{kg}$, etwa 70 bis $90 \%$ der Substanz wird an Plasmaproteine gebunden. In neutrophilen Granulozyten reichert sich Tigecyclin 
an. Die Elimination erfolgt überwiegend unverändert mit dem Stuhl; im Urin wurde nur etwa ein Drittel der verabreichten Dosis nachgewiesen. Die Eliminationshalbwertzeit beträgt etwa 42 Stunden, wobei große interindividuelle Unterschiede bestehen. Im Rahmen der klinischen Prüfung verursachte Tigecyclin signifikant häufiger gastrointestinale Nebenwirkungen als die Vergleichsantibiotika, Hautreaktionen traten dagegen seltener als in den Vergleichsgruppen auf.

Tigecyclin ist als eine Alternative bei komplizierten bakteriellen Hautinfektionen anzusehen, insbesondere wenn eine Allergie gegen andere Antibiotika bekannt ist. In klinischen Studien bei nosokomialer Pneumonie sowie bei Studien mit resistenten Erregern wurde bei den mit Tigecyclin behandelten Patienten eine numerisch höhere Mortalitätsrate als unter der Vergleichsmedikation beobachtet. Die Gründe hierfür sind unbekannt. Für andere Indikationen als komplizierte Haut- und Weichgewebsinfektionen und komplizierte intraabdominelle Infektionen ist Tyga$\mathrm{cil}^{\circledR}$ nicht zugelassen. Eine Anwendung in nicht zugelassenen Indikationen wird nicht empfohlen [41].

\section{Fazit}

\section{$\nabla$}

Eine Betrachtung der Antibiotika, die zur Behandlung einer MRSA-Infektion zur Verfügung stehen, zeigt exemplarisch, welche Eigenschaften eine antibiotisch wirksame Substanz für eine erfolgreiche Pharmakotherapie aufweisen muss. Insbesondere wird an mehreren Beispielen deutlich, dass der Nachweis einer in vitro-Aktivität zwar eine wichtige Voraussetzung für eine therapeutische Wirksamkeit ist, dass aber die für eine Therapieentscheidung notwendigen Erkenntnisse nur aus doppelblind-randomisierten klinischen Studien abgeleitet werden können. Die mögliche Toxizität der Arzneistoffe und ihr Interaktionspotenzial müssen in allen Fällen beachtet werden. Da sie sich zwischen den einzelnen Antibiotika deutlich unterscheiden, ergeben sich entsprechende differenzialtherapeutische Möglichkeiten. Bemerkenswert ist, dass Vancomycin auch nach 50 Jahren immer noch das Standardantibiotikum ist. Angesichts der abnehmenden Empfindlichkeit der Staphylokokken wären höhere Dosierungen sinnvoll, dies wird aber durch die dosisabhängig zunehmende Nephrotoxizität sehr eingeschränkt. Linezolid war in den Vergleichsstudien ähnlich wirksam, in einigen dem Vancomycin sogar überlegen. Bei diesem Oxazolidinon muss auf das hämatotoxische und neurotoxische Potenzial geachtet werden und die Möglichkeit der MAO-Inhibition.

Die klinischen Studien mit Daptomycin und anschließende gezielt durchgeführte in vitro-Experimente haben erkennen lassen, dass diese Substanz nicht bei pulmonalen Infektionen eingesetzt werden kann, weil sie durch Surfactant inaktiviert wird. Die nicht überzeugenden Therapieresultate mit Ceftobiprol bei Beatmungs-assoziierten Pneumonien und anschließende Subgruppenanalysen weisen auf die besonderen therapeutischen Probleme hin, die bei diesen Patienten bestehen. Insbesondere bei schwer kranken, jüngeren Patienten müssen Antibiotika hoch dosiert werden, um der häufig erhöhten renalen Clearance und dem erhöhten Verteilungsvolumen Rechnung zu tragen. Dies wurde anscheinend in der klinischen Prüfung nicht ausreichend berücksichtigt.

Die Behandlung von MRSA-Infektionen der tiefen Atemwege erscheint nach wie vor verbesserungsfähig. Mit der klinischen Prüfung von neueren antibiotisch wirksamen Arzneistoffen, wie Telavancin, Tedizolid und anderen, werden wir in den nächsten
Jahren neue Erkenntnisse gewinnen und in Zukunft vielleicht die notwendige weitere Verbesserung der Therapieresultate erreichen.

\section{Interessenkonflikt}

R. Stahlmann erhielt finanzielle Unterstützung für Forschungsprojekte bzw. Referenten- oder Beraterhonorare von den Firmen Bayer, Berlin Chemie, Chiesi und Merlion. Ein direkter Einfluss auf das Manuskript liegt nicht vor.

\section{Literatur}

1 Moellering RC Jr. MRSA: the first half century. J Antimicrob Chemother 2012; 67: 4-11

2 Lode HM. Clinical impact of antibiotic-resistant Gram-positive pathogens. Clin Microbiol Infect 2009; 15: 212-217

3 Arias CA, Murray BE. Antibiotic-resistant bugs in the 21st century - a clinical super-challenge. N Engl J Med 2009; 360: 439-443

4 Levine DP. Vancomycin: a history. Clin Infect Dis 2006; $42: 5-12$

5 Gastmeier P, Schröder C, Behnke M et al. Dramatic increase in vancomycin-resistant enterococci in Germany. J Antimicrob Chemother 2014; 69: 1660 - 1664

6 Lodise TP, Drusano GL, Butterfield JM et al. Penetration of vancomycin into epithelial lining fluid in healthy volunteers. Antimicrob Agents Chemother 2011; 55: 5507-5511

7 Svetitsky S, Leibovici L, Paul M. Comparative efficacy and safety of vancomycin versus teicoplanin: systematic review and meta-analysis. Antimicrob Agents Chemother 2009; 53: 4069-4079

8 Lodise TP, Lomaestro B, Graves J et al. Larger vancomycin doses (at least four grams per day) are associated with an increased incidence of nephrotoxicity. Antimicrob Agents Chemother 2008; 52: 1330-1336

9 Stahlmann R, Lode H. Pharmakokinetik von Antibiotika bei kritisch kranken und übergewichtigen Patienten. Dtsch Med Wschr 2014: (im Druck)

10 Bosso JA, Nappi J, Rudisill C et al. Relationship between vancomycin trough concentrations and nephrotoxicity: a prospective multicenter trial. Antimicrob Agents Chemother 2011; 55: 5475-5479

11 Cataldo MA, Tacconelli E, Grilli $E$ et al. Continuous versus intermittent infusion of vancomycin for the treatment of Gram-positive infections: systematic review and meta-analysis. J Antimicrob Chemother 2012; 67: $17-24$

12 Schmelzer TM, Christmas AB, Norton HJ et al. Vancomycin intermittent dosing versus continuous infusion for treatment of ventilator-associated pneumonia in trauma patients. Am Surg 2013; 79: 1185-1190

13 Gotfried MH, Shaw JP, Benton BM et al. Intrapulmonary distribution of intravenous telavancin in healthy subjects and effect of pulmonary surfactant on in vitro activities of telavancin and other antibiotics. Antimicrob Agents Chemother 2008; 52: 92 -97

14 Saravolatz LD, Stein GE, Johnson LB. Telavancin: a novel lipoglycopeptide. Clin Infect Dis 2009; 49: 1908 - 1914

15 Rubinstein E, Lalani T, Corey GR et al. Telavancin versus vancomycin for hospital-acquired pneumonia due to gram-positive pathogens. Clin Infect Dis 2011; 52: $31-40$

16 Corey GR, Kollef MH, Shorr AF et al. Telavancin for Hospital-acquired Pneumonia: Clinical Response and 28-Day Survival. Antimicrob Agents Chemother 2014; 58: 2030-2037

17 NN 2014, Vibativ, Zusammenfassung der Merkmale des Arzneimittels. www.ema-europa.eu

18 Long KS, Vester B. Resistance to linezolid caused by modifications at its binding site on the ribosome. Antimicrob Agents Chemother 2012; 56: $603-12$

19 Morales G, Picazo JJ, Baos E et al. Resistance to linezolid is mediated by the cfr gene in the first report of an outbreak of linezolid-resistant Staphylococcus aureus. Clin Infect Dis 2010; 50: 821 - 825

20 Locke JB, Morales G, Hilgers $M$ et al. Elevated linezolid resistance in clinical cfr-positive Staphylococcus aureus isolates is associated with cooccurring mutations in ribosomal protein L3. Antimicrob Agents Chemother 2010; 54: $5352-5355$ 
21 Mendes RE, Hogan PA, Streit JM et al. Zyvox Annual Appraisal of Potency and Spectrum (ZAAPS) Program: report of linezolid activity over 9 years (2004-12). J Antimicrob Chemother 2014; 69: 1582-1588

22 Locke JB, Finn J, Hilgers $M$ et al. Structure-activity relationships of diverse oxazolidinones for linezolid-resistant Staphylococcus aureus strains possessing the cfr methyltransferase gene or ribosomal mutations. Antimicrob Agents Chemother 2010; 54: 5337-5343

23 Moellering RC. Tedizolid: a novel oxazolidinone for Gram-positive infections. Clin Infect Dis 2014; $58: 1-3$

24 Clemett D, Markham A. Linezolid. Drugs 2000; 59: 815-827

25 Honeybourne $D$, Tobin $C$, Jevons $G$ et al. Intrapulmonary penetration of linezolid. J Antimicrob Chemother 2003; 51: 1431 - 1434

26 Nukui Y, Hatakeyama S, Okamoto K et al. High plasma linezolid concentration and impaired renal function affect development of linezolidinduced thrombocytopenia. J Antimicrob Chemother 2013; 68: $2128-2133$

27 Wunderink RG, Niederman MS, Kollef $M H$ et al. Linezolid in methicillinresistant Staphylococcus aureus nosocomial pneumonia: a randomized, controlled study. Clin Infect Dis 2012; 54: 621 -629

28 Chastre J, Blasi F, Masterton RG et al. European perspective and update on the management of nosocomial pneumonia due to methicillin-resistant Staphylococcus aureus after more than 10 years of experience with linezolid. Clin Microbiol Infect 2014; $20: 19-36$

29 Pucci MJ, Bush K. Investigational antimicrobial agents of 2013. Clin Microbiol Rev 2013; 26: 792 -821

30 File TM Jr, Wilcox MH, Stein GE. Summary of ceftaroline fosamil clinical trial studies and clinical safety. Clin Infect Dis 2012; $55: 173-180$

31 Poon H, Chang MH, Fung HB. Ceftaroline fosamil: a cephalosporin with activity against methicillin-resistant Staphylococcus aureus. Clin Ther 2012; 34: $743-765$
32 File TM Jr, Low DE, Eckburg PB et al. Integrated analysis of FOCUS 1 and FOCUS 2: randomized, doubled-blinded, multicenter phase 3 trials of the efficacy and safety of ceftaroline fosamil versus ceftriaxone in patients with community-acquired pneumonia. Clin Infect Dis 2010; 51: $1395-1405$

33 Casapao AM, Davis SL, Barr VO et al. A Large Retrospective Evaluation of the Effectiveness and Safety of Ceftaroline Fosamil Therapy. Antimicrob Agents Chemother 2014; 58: 2541 -2546

34 Lagacé-Wiens PR, Rubinstein E. Pharmacokinetic and pharmacodynamics evaluation of ceftobiprole medocaril for the treatment of hospital-acquired pneumonia. Expert Opin Drug Metab Toxicol 2013; 9: 789-799

35 Gonzalez-Ruiz A, Beiras-Fernandez A, Lehmkuhl H et al. Clinical experience with daptomycin in Europe: the first 2.5 years. . J Antimicrob Chemother 2011; 66: 912 -919

36 Seaton RA, Gonzalez-Ramallo VJ, Prisco V et al. Daptomycin for outpatient parenteral antibiotic therapy: a European registry experience. Int J Antimicrob Agents 2013; 41: 468-472

37 Silverman JA, Mortin LI, Vanpraagh AD et al. Inhibition of daptomycin by pulmonary surfactant: in vitro modeling and clinical impact. J Infect Dis 2005; 191: 2149-2152

38 Falcone $M$, Russo A, Venditti $M$ et al. Considerations for higher doses of daptomycin in critically ill patients with methicillin-resistant Staphylococcus aureus bacteremia. Clin Infect Dis 2013; 57: 1568-1576

39 Frampton JE, Curran MP. Tigecycline. Drugs 2005; 65: 2623-2637

40 Livermore DM. Tigecycline: what is it, and where should it be used? J Antimicrob Chemother 2005; 56: 611-614

41 Pharmacia, 2013. Fachinformation Tygacil ${ }^{\circledR}$

42 Rodvold KA, McConeghy KW. Methicillin-resistant Staphylococcus aureus therapy: past, present, and future. Clin Infect Dis 2014; 58 : $20-27$ 